\title{
Vortex ring propagation and interactions studies
}

\author{
K T IJAS HUSSAIN and T J S JOTHI* \\ Department of Mechanical Engineering, National Institute of Technology Calicut, Kerala 673601, India \\ e-mail: tjsjothi@nitc.ac.in
}

MS received 29 January 2017; revised 11 November 2017; accepted 12 January 2018; published online 18 June 2018

\begin{abstract}
This paper experimentally investigates the vortex ring propagation and interactions with thin cylindrical and flat surfaces. Dye-based visualization technique is adopted for the interaction studies. Vortex rings are generated from a circular nozzle of $19 \mathrm{~mm}$ diameter with the stroke length ratios (length of the fluid slug to nozzle diameter, $L_{N} / D_{N}$ ) of 1 to 5 , and ejection velocities in the range of 0.05 to $0.2 \mathrm{~m} / \mathrm{s}$. Vortex interaction studies are carried out with two different bodies; firstly, with the circular cylinders having the diameters of $0.2,0.6,1.5$ and $2.5 \mathrm{~mm}$, and secondly with a flat solid surface. Results indicate that the trails in the vortex ring start following at $L_{N} / D_{N}=4$. The influence of the initial velocity is found to be insignificant on the vortex ring diameter, however, found to depend on stroke length ratio. Vortex-cylinder interaction studies indicated that vortex velocity decreases with increase in cylinder diameter after the interaction. Reconnections of vortex rings are observed in lower cylinder diameter cases. In case of vortex ring interaction with the flat surface, stretching of the vortex core is observed leading to a considerable increase in the vortex ring diameter.
\end{abstract}

Keywords. Vortex ring; cylindrical surface; flat surface; bifurcation; vortex interactions.

\section{Introduction}

Vortex rings are formed when a slug of fluid is plunged into the surrounding fluid. It is a torus-shaped fluid structure that propagates in the ambient fluid with a certain velocity. Vortex ring patterns can be observed in smoke generators, explosions, volcanic eruption, etc. The practical applications of vortex rings are observed in industrial chimneys, effluents disposal in oceans/rivers, and so on. Further, among the biological perspective, the locomotion of aquatic creatures like jellyfish, salp, etc., generate vortex rings for propulsion. Numerous research works are carried out to understand the rich physics involved in the generation, propagation, and dissipation of vortex rings. The slug of fluid ejected from the nozzle rolls up to form a vortex ring. The fully developed vortex ring propagates at certain velocity followed by an azimuthal instability of the vortex ring core. Finally, the vortex ring breaks down and dissipate into the surrounding fluid. The schematic of vortex ring evolution is shown in figure 1. A vortex ring structure that is fully developed is majorly characterized by the ring diameter, vortex circulation and propagation velocity [1]. The vortex ring evolution depends on three parameters namely the nozzle diameter $\left(D_{N}\right)$, the length of the ejected fluid column $\left(L_{N}\right)$, and velocity of the ejected fluid $\left(U_{N}\right)$ as shown in figure 1 . Maxworthy [2] observed a decrease in the velocity during the propagation of vortex ring besides

*For correspondence the increase in volume due to the entrainment of the ambient fluid. During propagation, due to the viscous dissipation, vortex core losses the angular momentum and diffuse radially. This dissipation of vorticity causes a low pressure in the surrounding fluid and thus gets entrained into the vortex ring. The azimuthal instability of the vortex ring will trail after the propagation of vortex ring. The instability wave is observed to grow in amplitude, and develop at the direction of $45^{\circ}$ to the propagation of the ring. The ratio of the wavelength of azimuthal waves to the vortex core diameter $(c)$ is found to be approximately constant, $\lambda / c=2.24$. This instability makes the vortex core a wavy structure, and eventually break down as a turbulent vortex ring. Further, the experiments carried out on vortex rings are compared with the analytical results, and concluded that the model underestimates the experimental results $[1,2]$. The discrepancy was due to the maximum velocity at the nozzle edge at lower stroke length ratios $\left(L_{N} /\right.$ $\left.D_{N}\right)$. At higher stroke length ratios, the maximum velocity is at the center due to boundary layer growth. Further, the entrainment of the fluid surrounding the nozzle results in the overall reduction of the vortex ring circulation.

Gharib et al [3] studied the vortex rings using DPIV technique to examine the effect of stroke length ratio on vortex ring formation, and noticed the maximum circulation of a vortex ring at $L_{N} / D_{N}=4$. At this stroke length ratio, the pinch-off of the leading vortex ring from the trailing jet is observed, and this ratio is termed as the Formation Number. The formation number is seen to 


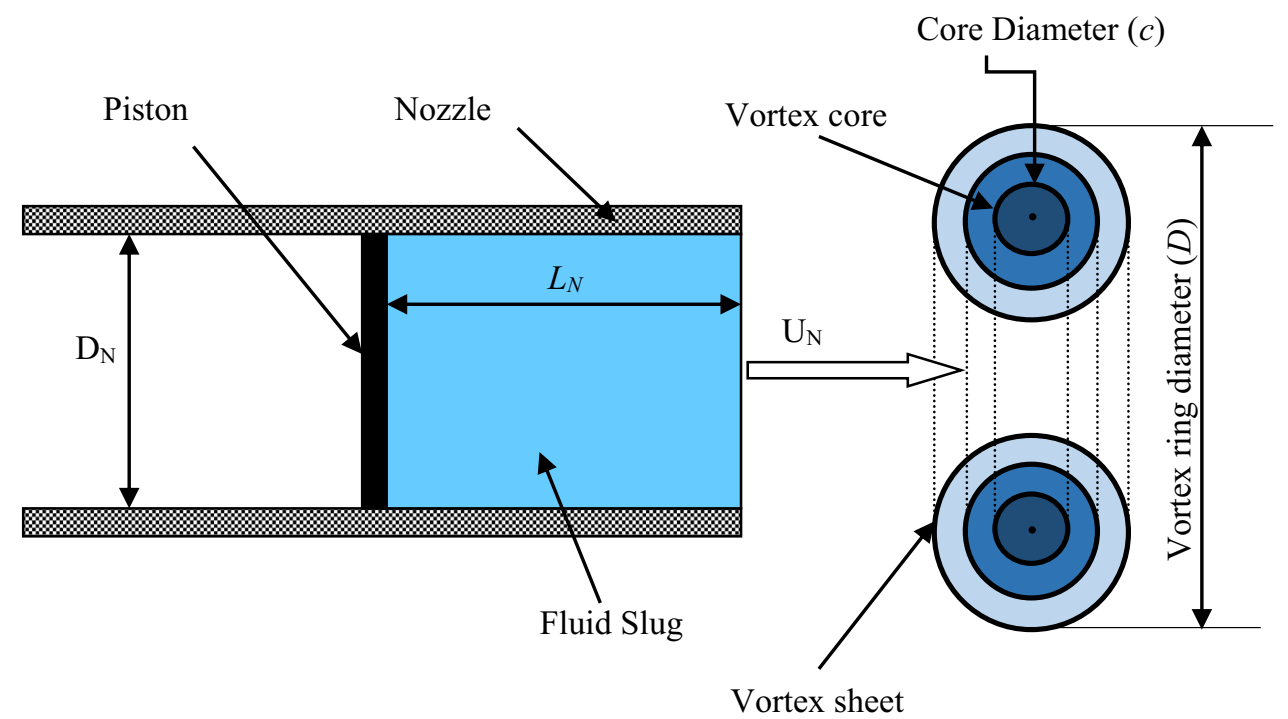

Figure 1. Schematic of the vortex ring generation.

depend on the initial boundary conditions, nozzle and piston profiles, and thus typically varies between 3.5 and 4.2. It is also observed that vortex rings generated at these formation numbers have the maximum energy. These findings are comparable with those of aquatic creatures which use the vortex rings for propulsion [4]. A non-linear correlation between dimensionless vortex ring diameter $(D /$ $D_{N}$ ) and stroke length ratio was presented by Maxworthy [5]. He showed that the vortex ring diameter has a weak dependence on the Reynolds number $(R e)$, and strong dependence on stroke length ratio. Considering the vortex ring diameter as the distance between the core centers, an empirical relation, Eq. (1) is proposed.

$$
\frac{D}{D_{N}}=1.18\left(\frac{L_{N}}{D_{N}}\right)^{0.25}
$$

The velocity of the viscous core vortex ring was given by Saffman [6] as shown in Eq. (2).

$$
U=\frac{\Gamma}{2 \pi D}\left\{\log \frac{4 D}{\sqrt{4 v t}}-0.558+O\left[\left(\frac{4 v t}{D^{2}}\right)^{\frac{1}{2}} \log \frac{4 v t}{D^{2}}\right]\right\}
$$

Lim and Nickels [7] studied the vortex ring in detail and showed that it consisted of vortex sheet and vortex core. The even distribution of the vorticity makes the vortex core a solid body rotation. Naitoh et al [8] introduced a swirling vortex ring to the surroundings and investigated its evolution using PIV technique. A strong vortex column is observed with a fluid termed as 'peeling off' that is seen to increase with the angular velocity of the rotating nozzle. Advaith et al [9] experimentally researched the interaction of vortex ring with the stratified interface using the highspeed shadowgraph and PIV. The vortex ring of varying strengths and surrounding fluid of varying degree of stratification are considered. The vortex ring penetration is found to depend on the Froude number where their relation is observed to be linear at lower Froude number.

Naitoh et al [10] carried out experiments to study the interaction of vortex rings with a circular cylinder placed in front of the ring. It was noted that the three factors namely, the Reynolds number, the ratio of cylinder diameter to core diameter $\left(d_{c} / c\right)$ and the ratio of core diameter to ring diameter controls the interaction. Their studies concluded that for $d_{c} / c \geq 0.025$, the interaction reduces the vortex ring propagation distance considerably. A numerical study on the working mechanism vortex ring - cut and reconnection was investigated by Ashurst and Meiron [11]. They explained the physics of core flattening and stretching, bridging of two vortices by cross-linking vortex lines for reconnection of the core. The analytical model proposed by Saffman [12] shows that the viscosity counters the vorticity of anti-parallel vortex core when they are in contact. This leads the fluid in the core to move axially away from the interaction point and thereby the ring is sheared and separated. Das et al [13] conducted experiments to study the interaction of vortex ring with a thin circular cylinder using PIV. By measuring the displacement of the streamlines in the flow field, the vortex core circulation, core diameter, vortex ring diameter, etc. are characterized. Kontis et al [14] carried out interaction experiments with different objects and explained that the interaction of vortex ring with different objects created different flow phenomenon due to the three-dimensional and time-dependent properties of the vortex ring. The upper and lower portions of the ring bend over the cylinder while the side portions expand outwards and the ring maintain its filament structure upon stretching in various directions during the interaction. Ren 
et al [15] numerically investigated the impinging of the vortex ring with the circular cylinder using large eddy simulation. The evolution dynamics of secondary, tertiary, hairpin, and small-scale vortices during the interaction is discussed. Musta [16] experimentally investigated the vortex ring past a thin cutting plate. For the cutting plate below the centerline, the vortex ring dispersed as three rings, one in the direction same as the primary vortex ring, and the other two in the directions perpendicular to the cutting plate. The vortex structure is found to be circulating when the vortex ring is cut up to the ring axis.

Yamada et al [17] conducted experiments with smoke visualization to study the vortex interaction near the wall. The observations concluded that the rebounding of primary vortex ring was due to the wall boundary layer separation. The boundary layer separation and secondary vortex generation during the interaction of vortex ring with a solid surface were depicted by Lim et al [18]. They conducted two experiments for explaining the phenomenon. First was the interaction of a vortex ring with a solid boundary, and the second set of experiments was on head-on collision of two identical vortex rings. The rebounding and secondary vortex ring generation is observed for the first set of experiments, which led to the conclusion that the secondary vortex ring is due to the boundary layer separation at the wall. Walker et al [19] conducted experiments to study the vortex ring interaction with a solid surface for a wide range of Reynolds numbers. At lower Reynolds number, the boundary layer separation and secondary vortex ring were absent due to insufficient circulation. The rebounding and trailing vortex ring was observed due to the boundary layer separation at higher Reynolds number. The trailing vortices formation was found to depend on stroke length ratio and Reynolds number. Cerra and Smith [20] conducted experiments for examining the breakdown of laminar vortex ring during its impact on solid surfaces. In the vicinity of the surface, the vortex ring is observed to be turbulent due to the rapid dispersion of ring vorticity to the surrounding fluid. A study on vortex ring interaction with an inclined wall is conducted by Lim [21] to explain the stretching mechanism closer to the wall, and compression of the ring portion away from the inclined wall.

Mujal-Colilles et al [22] conducted experiments to investigate the vortex rings interaction with solid and permeable objects using PIV. Permeability changed the stretching of the rings with the generation of secondary vortices. Importantly, in permeable walls, a slip boundary condition is observed that influence the secondary vortex. New et al [23] experimentally studied the interaction of vortex rings of different Reynolds number with inclined surfaces in the range of $30^{\circ}$ to $75^{\circ}$. Near the inclined surface at larger angles, size of primary vortex ring reduced rapidly, however, became larger in the far field. Formation of the secondary vortex-rings is noticed in the near field, and later interacted with the primary vortex ring in the far field. Additional vortex cores entwining the primary vortex referred as a "bi-helical vortex" is observed. Xu and Wang [24] investigated the impinging of vortex rings generated at different stroke lengths on a solid wall. During the interaction, secondary vortex structures are observed near the wall at higher stroke lengths. Interestingly, this secondary vortex prevents the accumulation of the impinging vortex rings on to the solid wall. At lower stroke lengths, a largescale spiral vortex ring is observed near the wall region that is seen to have a lower decay rate in the radial direction.

It is viable from the literature that the vortex flow mechanisms are the basic building blocks of a complex fluid flow phenomenon and thus creating more interest among the fluid mechanics researchers in understanding the underlying mechanism. This article primarily concentrates on the fundamental study of vortex ring propagation, evolution and its interaction with obstructions such as cylindrical and flat solid surfaces. Albeit numerous studies exist in archival, they are extensively studied individually on the above-mentioned topics. A lacuna is observed in the understanding of vortex ring propagation and its interaction phenomenon with and without obstruction under a single platform as a whole. Thus the present paper collectively investigates the characteristics of free vortex ring propagation, vortex ring interaction with the cylindrical surface, and vortex ring interaction with the flat surface. The vortex ring diameters and its propagation velocity are examined for the above three mentioned mechanisms, and are compared.

\section{Experimental set-up}

Dye-based flow visualization technique is adopted for this study. Experiments are carried out in an in-house fabricated set-up as shown in figure 2 a considering water as the working fluid to generate the vortex rings. The set-up consists of a nozzle arrangement connected to a fluid tank that acts as the surrounding. A nozzle is fabricated using mild steel material with the diameter of $19 \mathrm{~mm}$. The vortex ring is generated by ejecting the fluid slug from the nozzle, which is performed using a pneumatically operated and controlled piston-cylinder mechanism. A DC compressor having the rating of $12 \mathrm{~V}$ and 7.2 Amps is used for the pneumatic operations. The fluid tank having the dimensions of $1.0 \times 0.3 \times 0.4 \mathrm{~m}^{3}$ is made of $4 \mathrm{~mm}$ thick transparent Poly Methyl MethAacrylate (PMMA) sheets. The on-off of vortex ring flow is controlled using the solenoid valve connected to the set-up. Methylene blue alkaline dye is used for visualization of vortex rings. The vortex rings are generated with the stroke length ratios in the range of $1 \leq L_{N} / D_{N} \leq 5$ and ejection velocities in the range of 0.05 to $0.2 \mathrm{~m} / \mathrm{s}$. The Reynolds numbers considered for the current experiments are in the range of 1200 to 2500 that are obtained by varying the flow velocity. The images of vortex rings are captured using CANON 1200D camera at the 
(a)

\section{Dye storage}

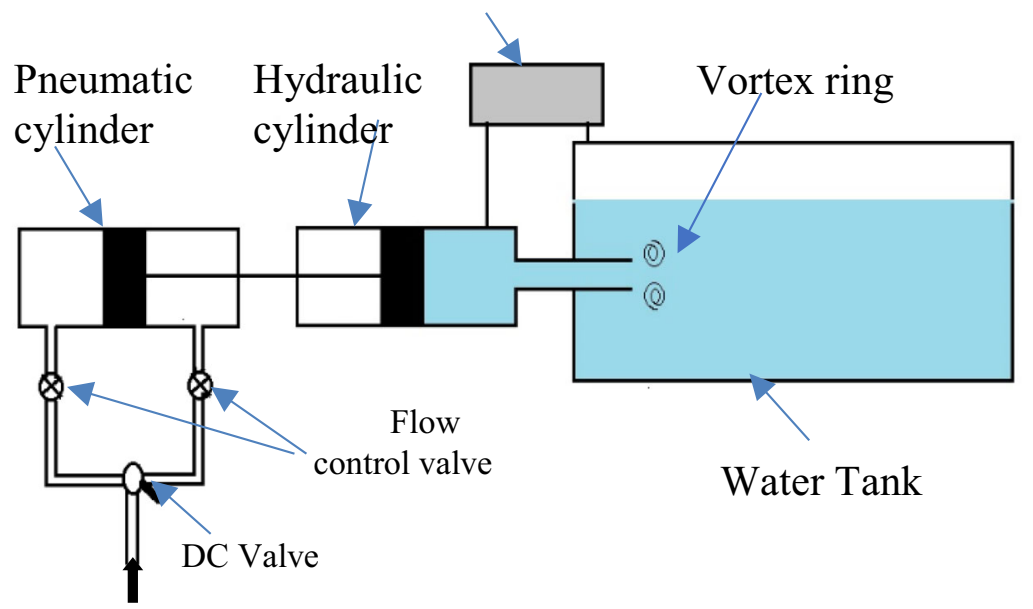

Compressed air

(b)

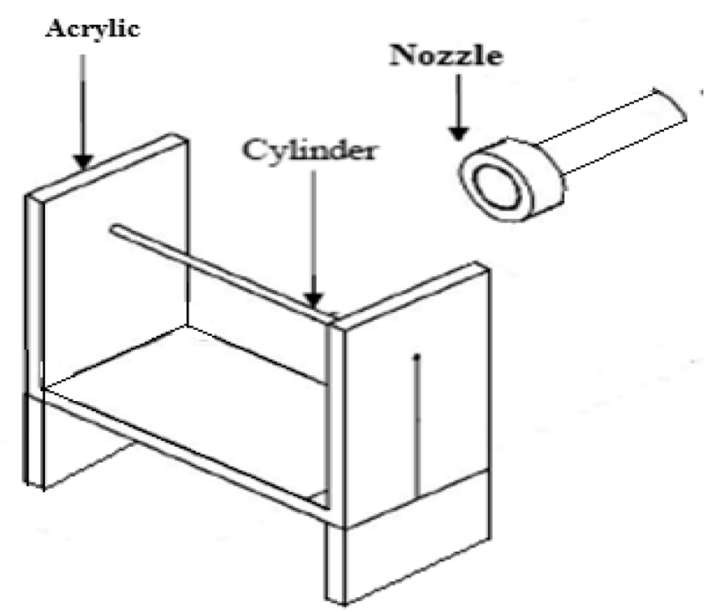

(c)

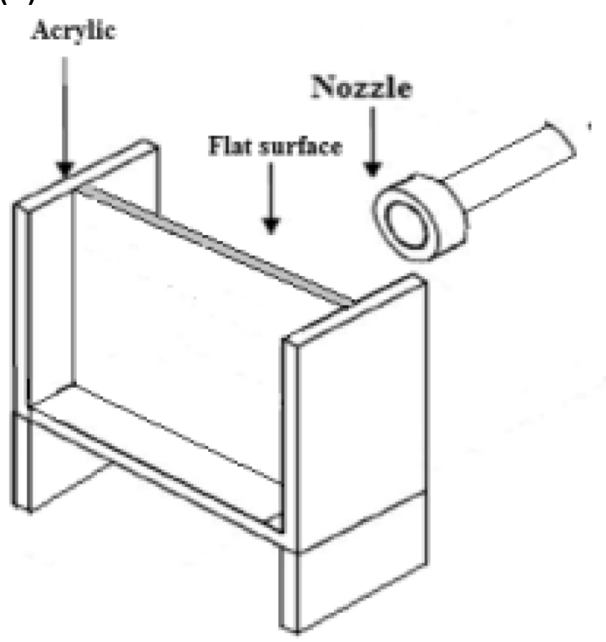

Figure 2. Schematic of the (a) vortex ring generator setup, (b) the interaction of vortex ring with the cylinder and (c) the interaction of vortex ring with flat surface.

resolution of $1920 \times 1080$ pixels. The images are processed using the software, and the linear dimensions in the image are within the uncertainty range of \pm 20 pixels that is equivalent to $\pm 2 \mathrm{~mm}$.

Initial studies are carried with free vortex ring propagation at different Reynolds numbers. The second sets of experiments are carried to investigate the vortex ring interactions with circular cylinders placed perpendicular to the ring propagation. The diameters of the various cylinders considered are 0.2, 0.6, 1.5 and $2.5 \mathrm{~mm}$. The Reynolds number and stroke length ratio chosen for this study are 1400 and $L_{N} / D_{N}=1$, respectively. The cylinders are placed at a distance of $5 D_{N}$ from the nozzle exit (figure $2 \mathrm{~b}$ ). The third set of experiments are carried out to study the interactions of a vortex ring with a flat surface made of PMMA sheet having a thickness of $5 \mathrm{~mm}$, placed at a distance of $5 D_{N}$ from the nozzle exit (figure $2 \mathrm{c}$ ).

\section{Results and discussion}

\subsection{Vortex ring}

Figure 3 shows the instantaneous images of the vortex ring generated at different stroke length ratios at the Reynolds number of 1700 . At lower stroke length ratio $\left(L_{N} / D_{N}=1\right)$, the volume of fluid slug in the nozzle is less, and thus the vortex generated has got a perfect ring structure. The distortions in the ring structure are evident at higher stroke lengths of $L_{N} / D_{N}>2$. Interestingly, at $L_{N} /$ $D_{N}=4$ and 5, vortex fluid trails the vortex rings that were not observed at lower stroke lengths. This is due to the fact that at higher stroke lengths, a surplus fluid ejected from the nozzle is unable to accommodate itself in vortex circulation thus forming the trails behind the vortex ring. While at lower stroke lengths, the volume of fluid ejected 

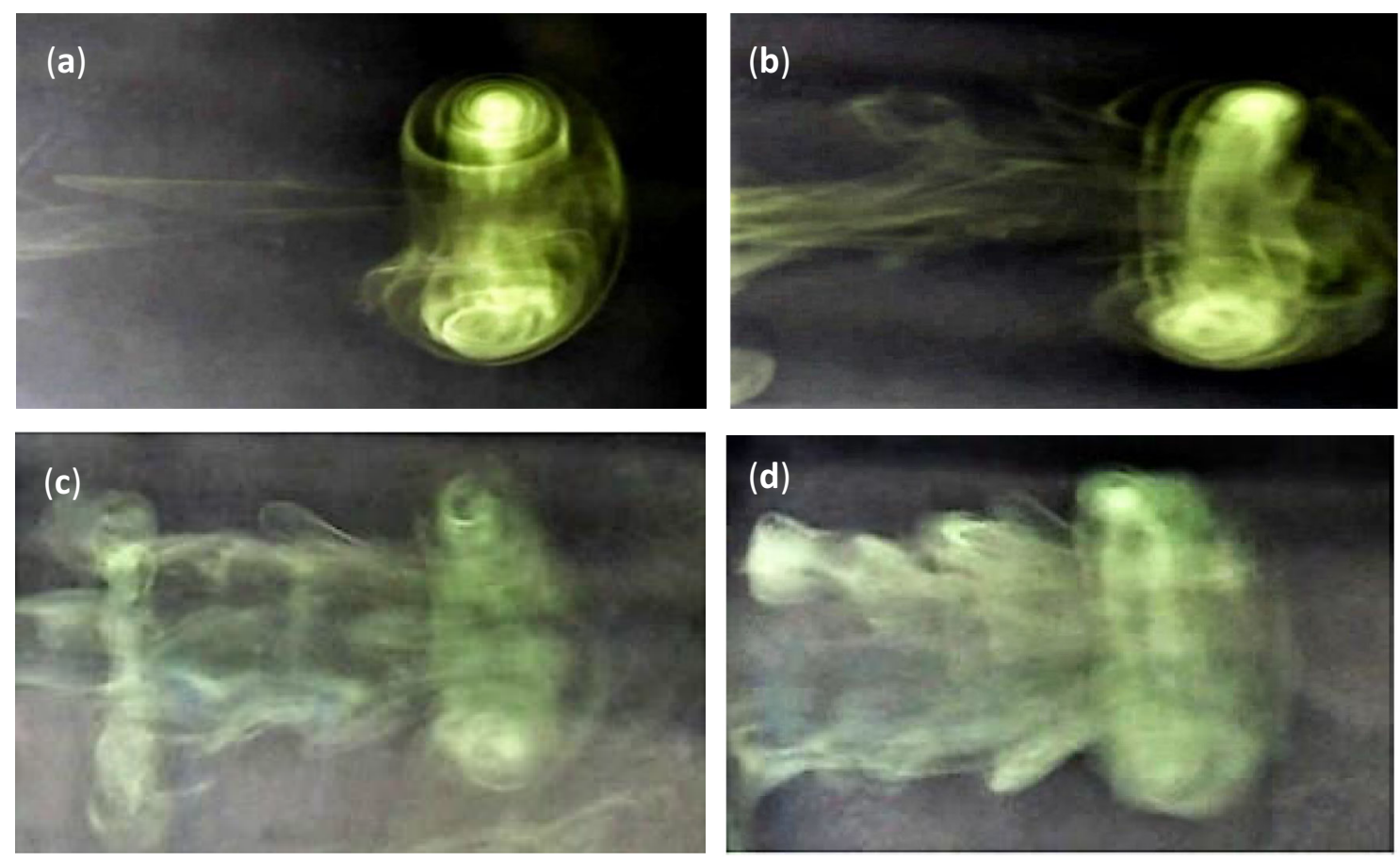

Figure 3. Vortex ring generated at stroke length ratios $\left(L_{N} / D_{N}\right)$ of (a) 1.0 , (b) 3.0, (c) 4.0 and (d) 5.0 at $R e=1700$.

completely circulates within the vortex ring. It can be corroborated that the maximum circulation of the vortex ring can be achieved at $L_{N} / D_{N}=4$, beyond which the circulation will remain constant nevertheless with the increase of volume ejected [2]. Further increase in the stroke lengths leads to the larger vortex fluid trails, thus triggering the turbulence, making the vortex ring completely chaotic. The following additional insights are noted from a vortex ring generated at a stroke length ratio of $L_{N} / D_{N}=1$. Figure 4 shows the location of vortex ring from the nozzle exit at different time intervals for $R e=1700$. It is observed from the figure that the vortex ring diameter increases with its propagation. This aspect is illustrated in figure 5 where the dimensionless vortex ring diameter $\left(D / D_{N}\right)$ is plotted with the axial distance. It is evident from the figure that the vortex ring diameter increases while propagation which is similar to the results observed by Maxworthy $[2,5]$. The reason is depicted to the entrainment of surrounding fluid into the vortex ring thus increasing the volume of the ring along the propagation, leading to an increase of ring diameter. The ring diameter increases approximately by $3 \%$ for the propagation distance of $4 D_{N}$. Figure 6 shows the location of the vortex ring with time. Initially, the gradient, which represents the velocity of the vortex ring, is found to be larger $(\approx 1.08)$, and subsequently gets smaller $(\approx 0.68)$ at higher intervals indicating the decrease in the vortex ring velocity with distance. Since the volume of the vortex ring increases with propagation as seen in figure 4, the decrease in its velocity is observed to conserve the momentum.
Figure 7 shows the variation of the dimensionless vortex ring diameter with stroke length ratios at $x$ / $D_{N}=5$ at different Reynolds number. The stroke length is limited to $L_{N} / D_{N}=4$ since the trailing effects dominate beyond this value. It is noted from figure 7 that the ring diameter increases with increase in stroke length at all the Reynolds numbers. It is due to the fact that the ejected fluid has higher volume for larger stroke lengths, and thus gets included in the circulation of the vortex ring thereby increasing the vortex ring diameter. However, there is no considerable variation in the ring diameters with respect to the Reynolds number. It is thus concluded that the vortex ring diameter is a sole function of stroke ratio. The relation between dimensionless vortex ring diameter and the stroke length ratio is given in Eq. (3).

$$
\frac{D}{D_{N}}=1.7\left(\frac{L_{N}}{D_{N}}\right)^{0.22}
$$

The obtained trend from the current study is in congruence with Eq. (1) suggested by Maxworthy [5], however, with a small deviation in the power of Eq. (3). This may be due to the fact that the present study considered the entire ring diameter (figure 1) for obtaining Eq. (3) while the centre to centre distance is considered as the ring diameter by Maxworthy [5]. The relationship between the dimensionless vortex core diameters $\left(c / D_{N}\right)$ with stroke length ratio at different Reynolds numbers is studied and plotted in figure 8 . The core diameter is measured at the location of $x / D_{N}=5$ from the nozzle exit. The core 

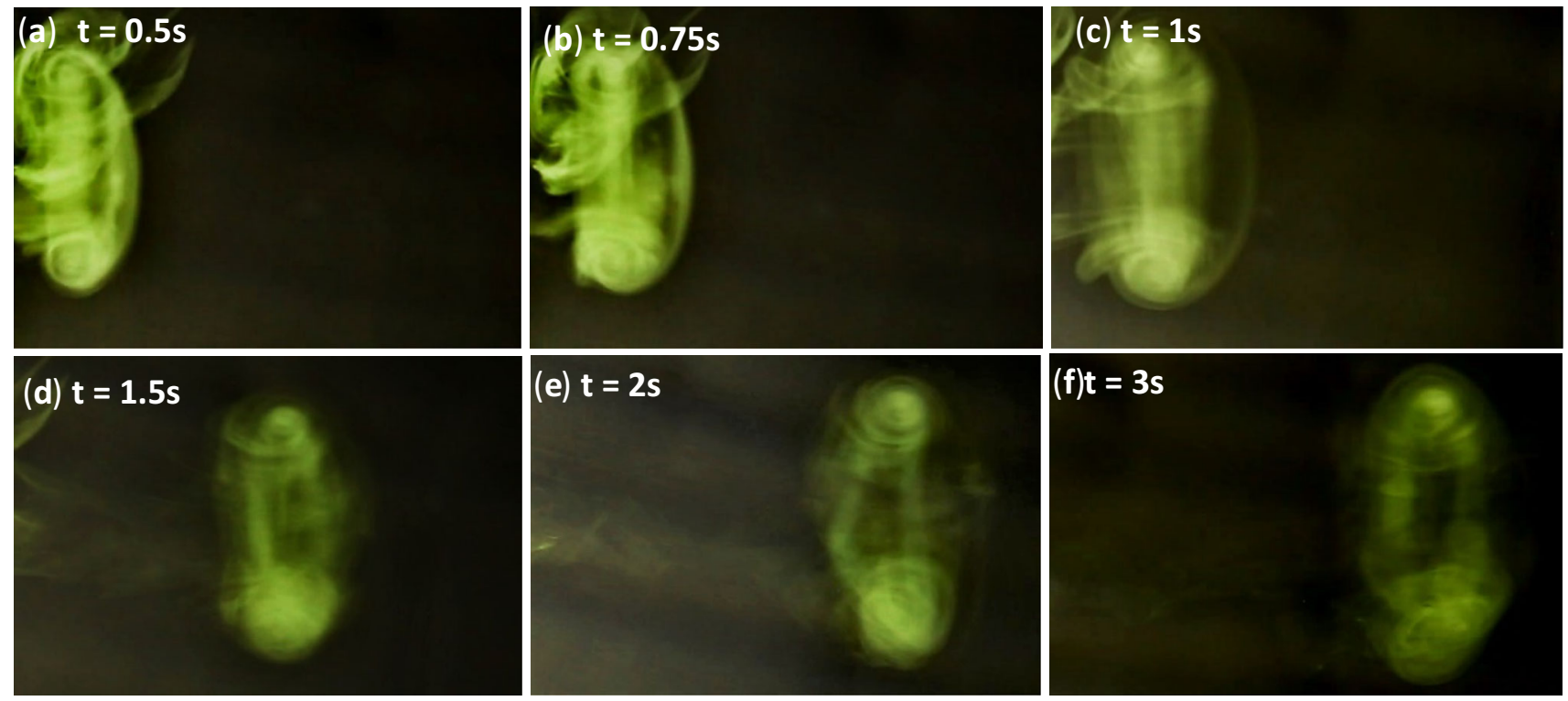

Figure 4. Vortex rings along the axial locations at different propagation time shown for vortex ring generated at $R e=1700$ and $L_{N} /$ $D_{N}=1$.

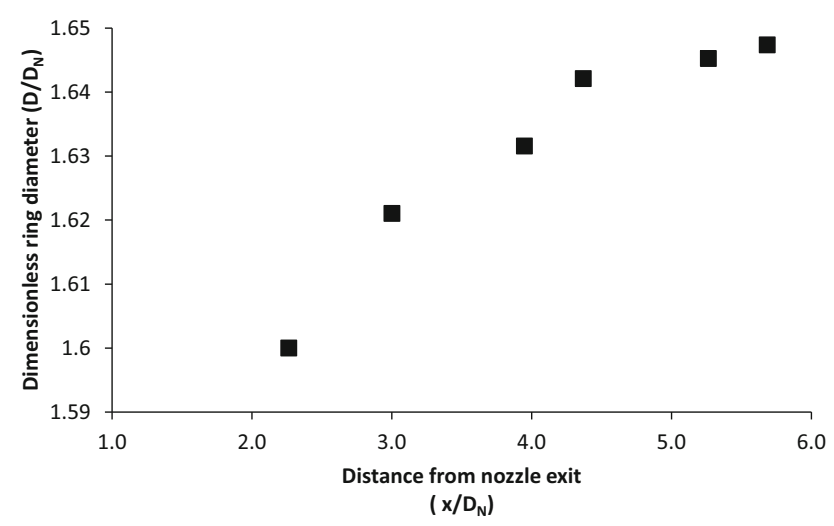

Figure 5. Variation of vortex ring diameter with axial distance at $R e=1700$ and $L_{N} / D_{N}=1$.

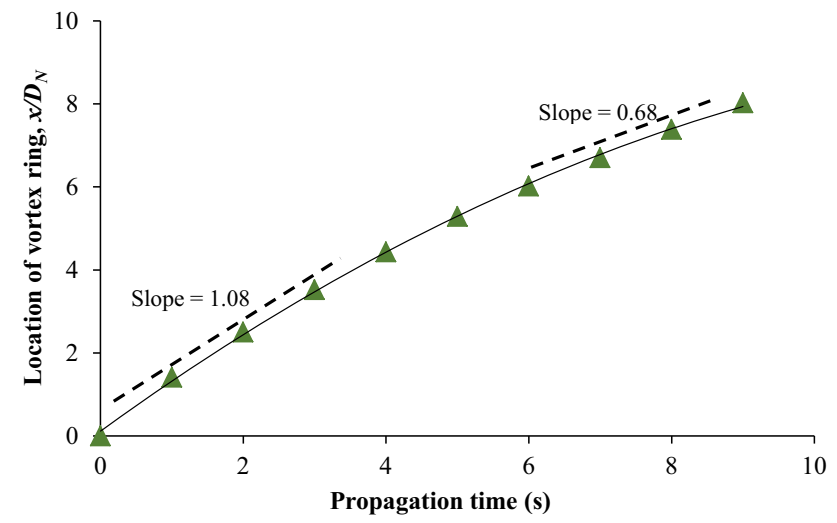

Figure 6. Location of the vortex ring with time at $R e=1700$ and $L_{N} / D_{N}=1$.

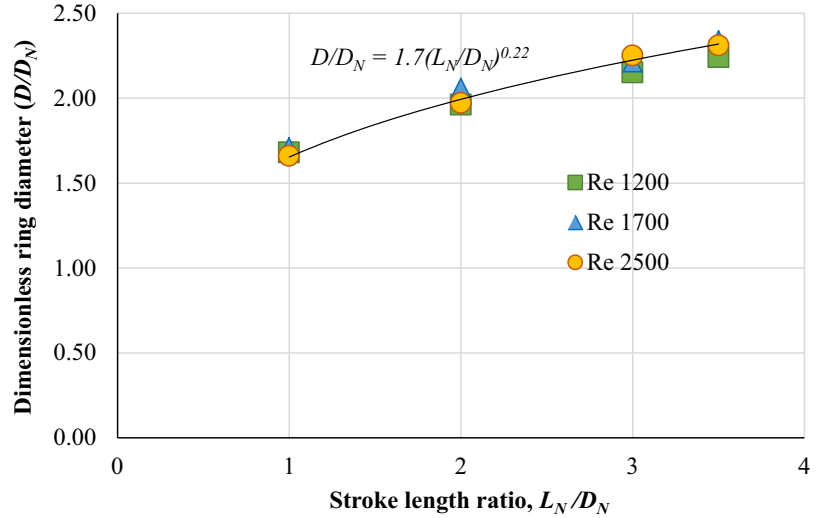

Figure 7. Variation of vortex ring diameter with stroke length ratio for different Reynolds numbers at $x / D_{N}=5$.

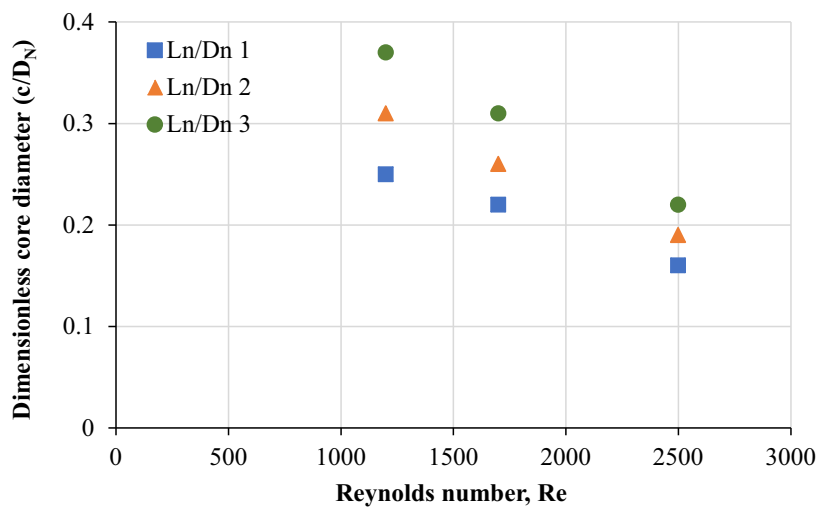

Figure 8. Variation of core diameter with the Reynolds number for different stroke length ratios at $x / D_{N}=5$. 

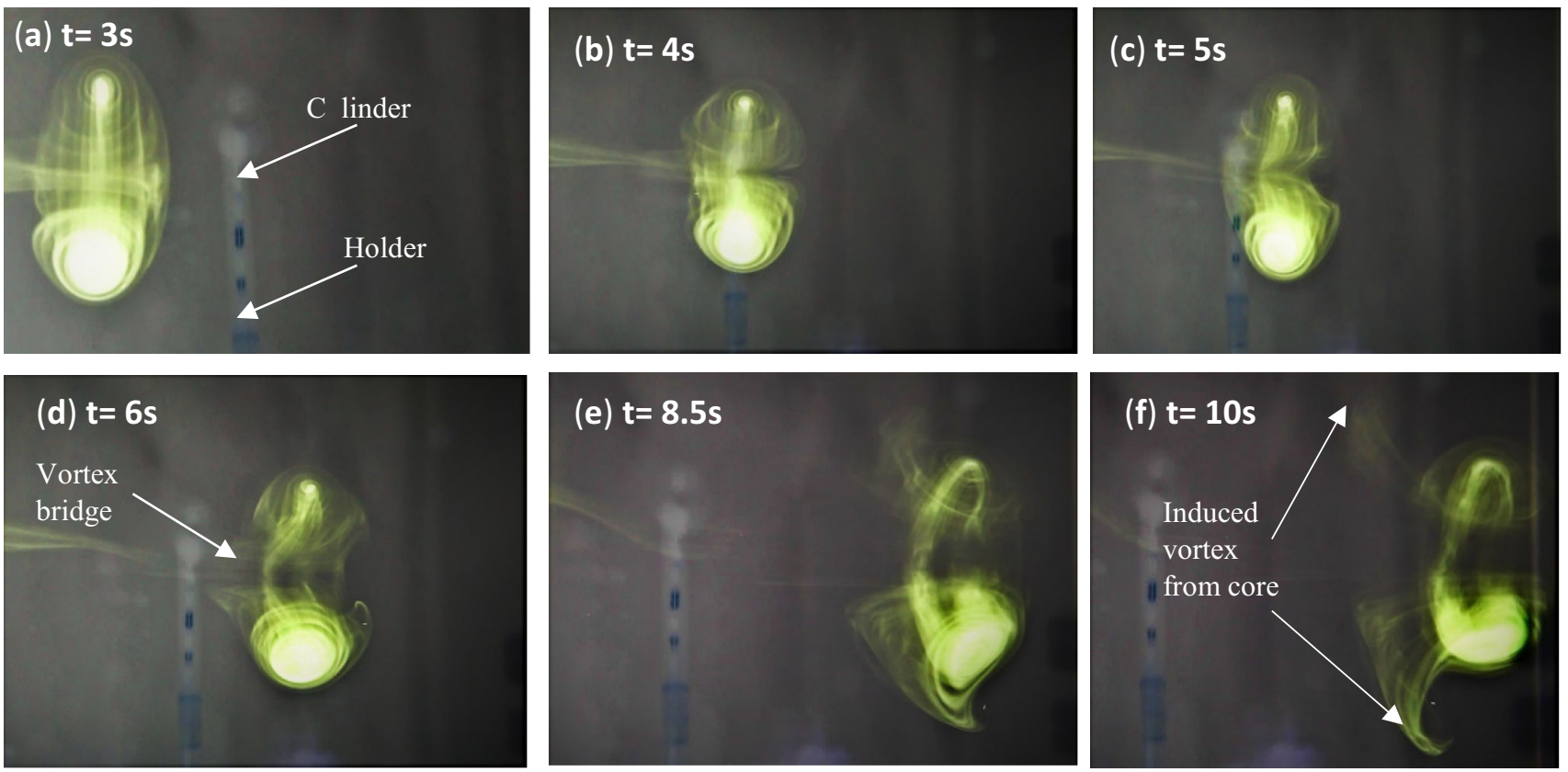

Figure 9. Interaction of vortex ring with cylinder of $0.2 \mathrm{~mm}$ diameter at different times at $R e=1400$.
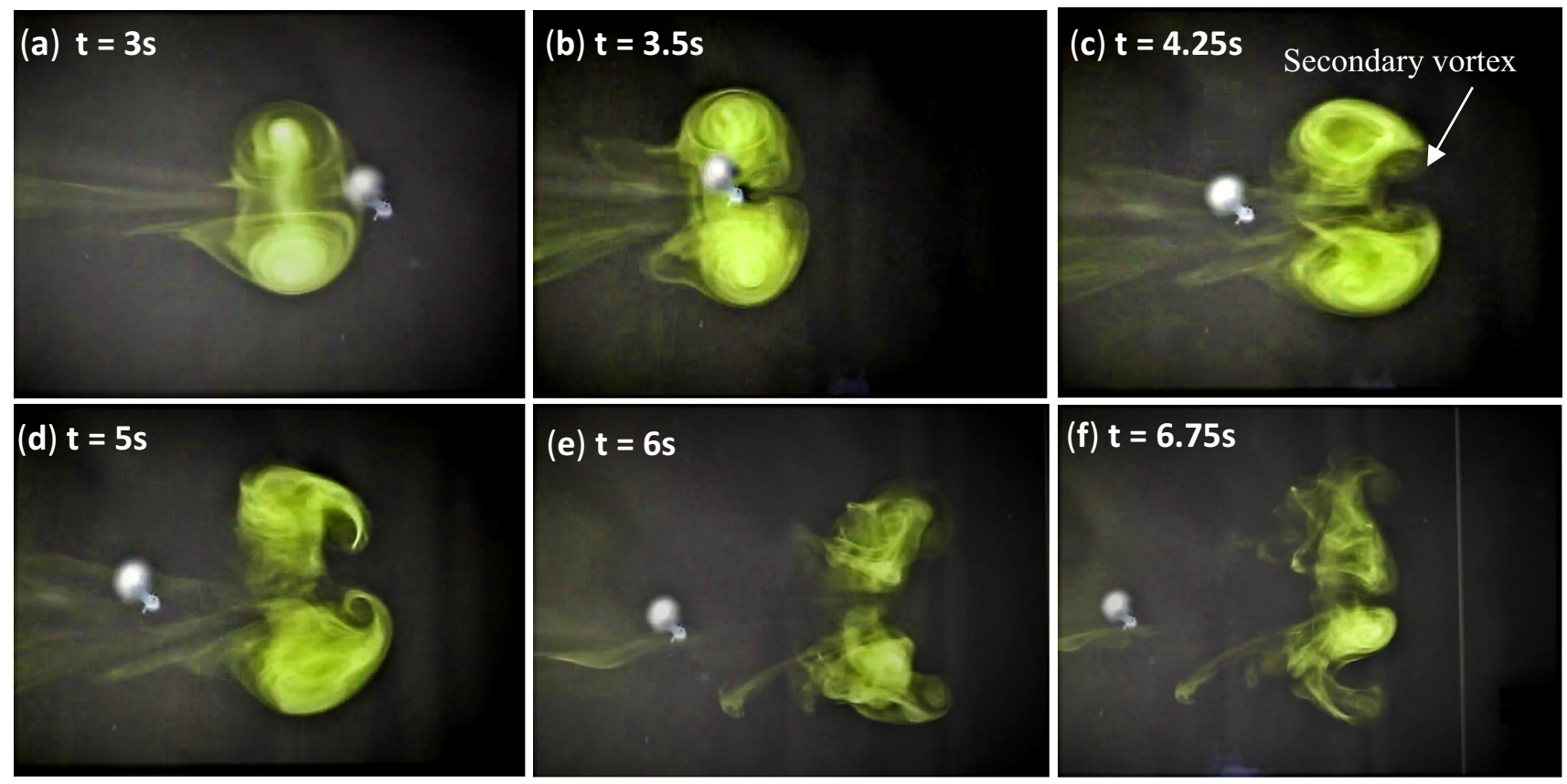

Figure 10. Interaction of vortex ring with a cylinder of $1.5 \mathrm{~mm}$ diameter at different times at $R e=1400$.

diameter is observed to increase with an increase in stroke length for an earlier reason stated. However, the major observation is the drastic reduction in the core diameter with an increase in the Reynolds number. The increase in the circulation of the vortex ring at higher Reynolds number may be the possible reason leading to smaller vortex core diameter. Thus, the vortex core diameter is found to depend on both the parameters namely the stroke length and the Reynolds number. These observations are in good agreement with the results obtained by Adhikari [25] where the Reynolds numbers considered were between 1000 and 1800 . 


\subsection{Vortex ring and cylindrical surface interaction}

This section presents the results obtained from the interaction of vortex rings with the cylindrical surfaces at an axial distance of $x / D_{N}=5$. The Reynolds number of the vortex ring considered is 1400 generated at $L_{N} / D_{N}=1$. The different stages of vortex interaction with a cylinder of $0.2 \mathrm{~mm}$ diameter are shown in figure 9. It is noticed that the ring passing through the cylinder is bifurcated due to the presence of cylinder. The vortex region in the vicinity of the cylinder slows down, and rest of the region propagates with the same velocity, thus creating a pressure difference between these two regions. This results in stretching (figure 9c) and division into two rings (figure 9d), followed by subsequent reconnections which are similar to the results observed by Saffman [12]. During the stretching of vortex rings, the counter-rotating vortices adjacent to the cylinder form a bridge-like trailing structure as shown in figure 9d. Further, a pair of counter-rotating secondary vortex is observed that moves radially away from the vortex ring (figure 9f). Similarly, vortex ring interaction with $0.6 \mathrm{~mm}$ diameter cylinder is investigated, and the results are found to be in parallel with the previous case. Figure 10 shows the vortex ring interactions with the cylindrical surface of $1.5 \mathrm{~mm}$ diameter. Here, after the bifurcation, the vortex core is deformed to an asymmetric shape (figure 10d) with an introduction of secondary vortices which was unlikely at smaller diameter surfaces, where the core shape was maintained. Further, it is evident from the figure $10(\mathrm{~b})-(\mathrm{d})$ that the secondary rings are generated from the sides of the cylinder as the passage of vortex ring. It is presumed that the generation of secondary vortices is due to the shear between the vortex ring and the cylinder [10]. The possible reconnection of the vortex ring is observed in this case however with a drastic distortion in the vortex core. These vortex ring interaction images obtained are comparable with those of Adhikari [25] where the experiments were carried out for the flow past a cylinder of $1.86 \mathrm{~mm}$ diameter. Similar experiments were carried out with a $2.5 \mathrm{~mm}$ diameter concluded the formation of asymmetric vortex rings. However, the reconnections of the bifurcated rings are not observed, and the vortex core turns out to be chaotic and dissipates instantly. The variation of dimensionless vortex ring diameter with the interaction of cylinders of various diameters is plotted in figure 11 . Until the distance $x / D_{N}=5$, the vortex ring behaves as a free vortex ring as described in the previous section 3.1. Subsequent to interaction, the vortex rings diameter is seen to suddenly increase with the axial length. Much increase in the ring diameter is observed in the case of $1.5 \mathrm{~mm}$ diameter cylinder due to the larger radial stretching, compared to the lower diameter cylinders. Figure 12 shows the location of the vortex ring with time. Prior to the interaction, the velocities of the all the vortex rings are similar. However, after the interaction, vortex ring past the lowest cylinder has the highest velocity. Compared to

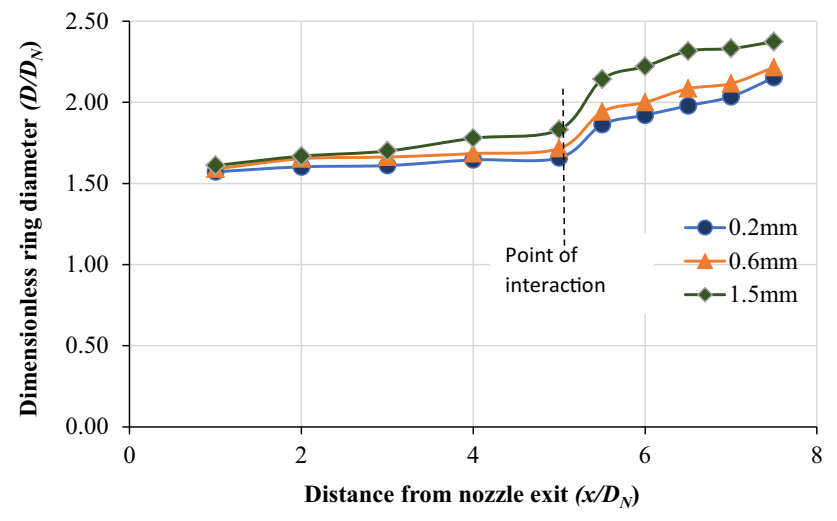

Figure 11. Variation of vortex ring diameter along its propagation during the interaction with cylinders of different diameters.

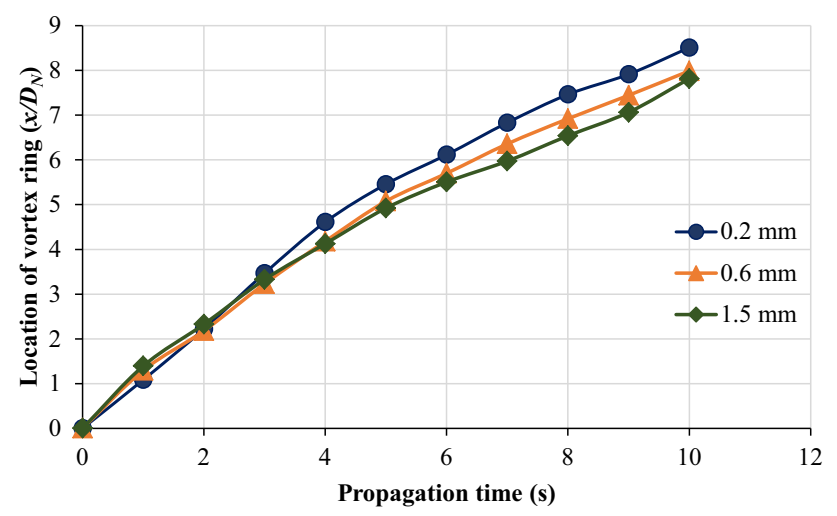

Figure 12. The locations of the vortex rings at different propagation times during the interaction with cylinders of different diameters.

the velocity prior to the interaction, the velocity after the interaction is found to be lower.

\subsection{Vortex rings and flat surface interaction}

In this study, the vortex ring interaction with the flat solid surface placed at a distance of $x / D_{N}=5$ from the nozzle exit is investigated. The vortex rings are generated with the stroke length ratio of 1 , and at the Reynolds numbers of 600,1400 and 2300. The stages of vortex ring interaction with the flat surface at the Reynolds number of 600 is shown in figure 13. The vortex ring diameter is seen to considerably increase with interaction (figure $13 \mathrm{c}-\mathrm{d}$ ) due to the stretching of the vortex core. Finally, the vortex rings completely dissipates into the surrounding fluid. This increase in the diameter is because of the obstruction created by the flat plate that restricts the vortex circulation leading to the fluid dissipation in the radial direction. Figure 14 shows the vortex interaction with the flat plate at the Reynolds number of 1400 . The higher velocity of the vortex ring leads to the formation of secondary vortices 

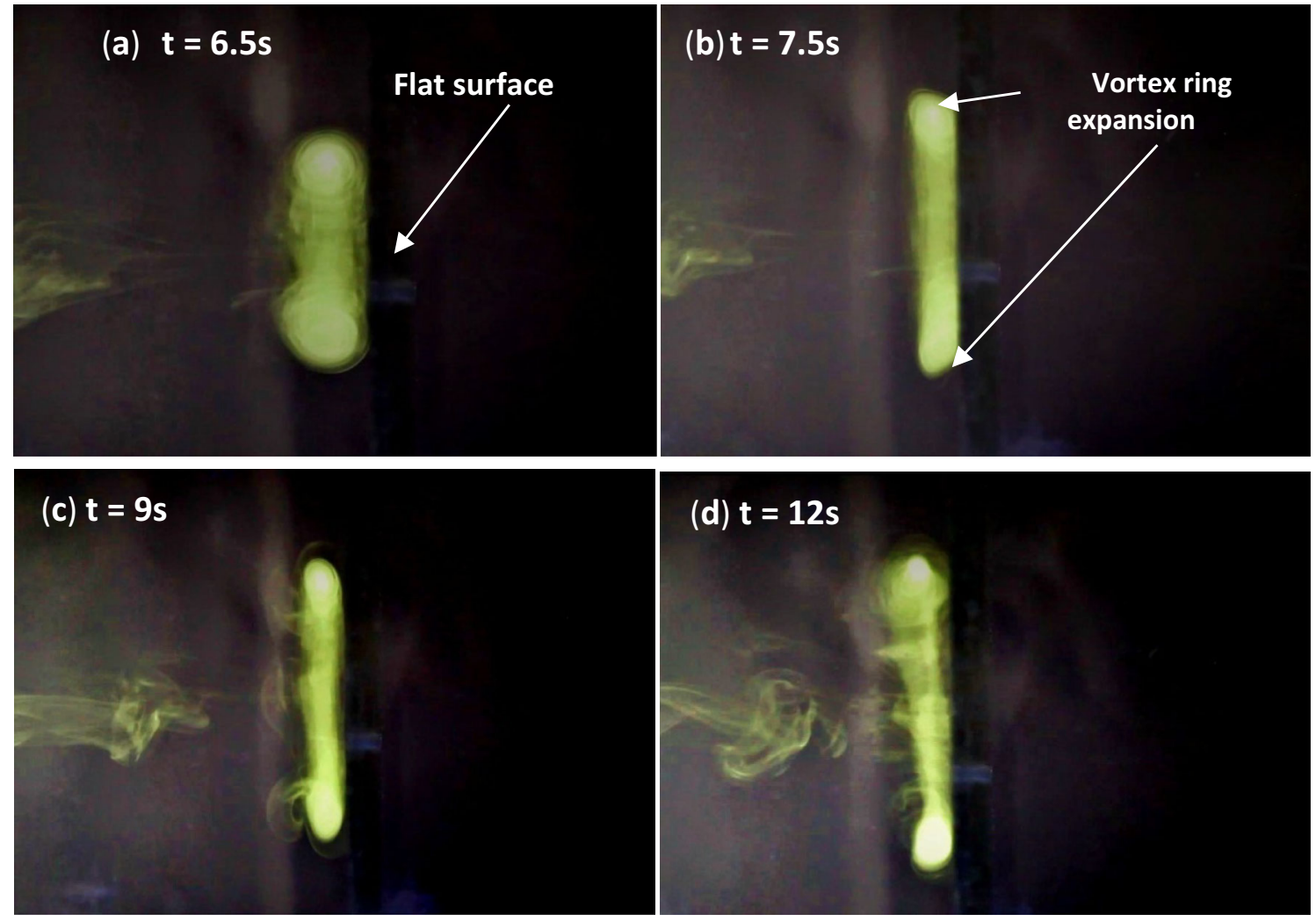

Figure 13. Interaction of vortex ring with a flat plate at different times for $R e=600$.
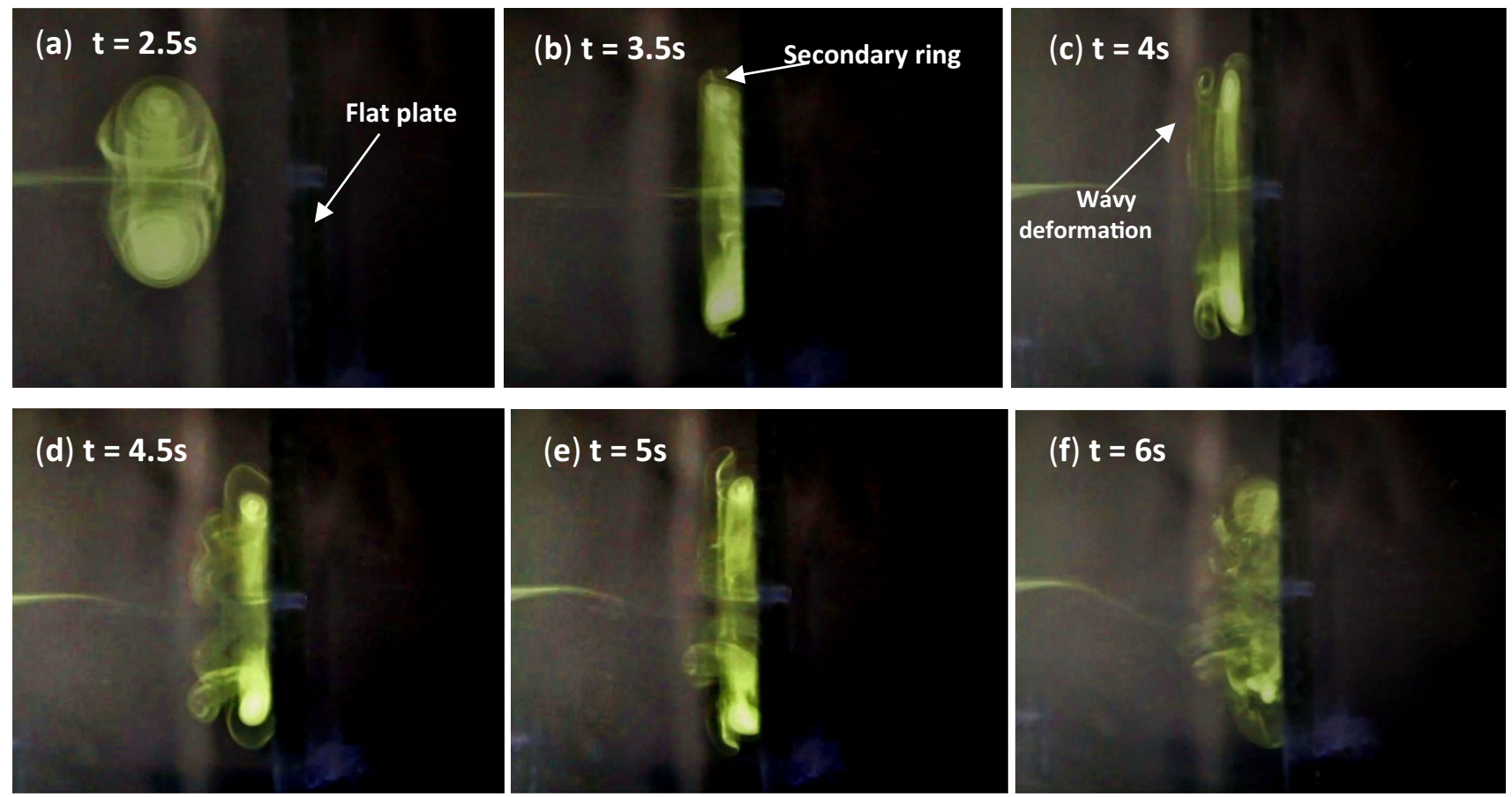

Figure 14. Interaction of vortex ring with the flat plate at different times for $R e=1400$. 
rolling up from the lee sides of primary vortex ring after the interaction (figure 14b). The formation of similar secondary vortices is observed by Lim et al [21], and the boundary layer separation under the vortex core is seen as the reason behind the same [19]. The secondary vortex ring has circulation in opposite direction as observed in figure 14(c) and (d). Further, due to the secondary vortices, the primary vortex ring is seen to rebound from the surface for few moments (figure 14d). The interaction between the secondary vortex ring and the rebounding primary ring is expected to initiate a wavy disturbances leading to dissipation of vortex ring completely. Further increase in the Reynolds number will cause the above events in a reduced time interval and destruction of the primary ring occurs all of a sudden. The variation in dimensionless vortex ring diameter during interaction with a flat surface is shown in figure 15 for different Reynolds numbers. It is observed that the vortex ring at higher Reynolds number attained the maximum ring diameter in short duration, while the lower Reynolds number vortex ring attained at higher duration. However, the maximum diameter obtained is the same at all the Reynolds numbers.

Figure 16 shows the growth in the vortex ring diameter with the propagation time for different interaction cases, and compared with that of a free vortex ring. The comparison was made for the vortex rings generated at $L_{N} /$ $D_{N}=1$ and $R e=1400$. The vortex ring growth is shown with respect to time due to the spatial restriction created by the presence of flat plate at $x / D_{N}=5$. From figure 16 , it is observed that up to the interaction point of $x / D_{N}=5$, the growth in the vortex ring diameter is similar in all three cases. During the interaction with flat plate, the vortex ring grows at higher rate followed by the growth due to the cylindrical surface. Noticeably, the free vortex rings growth is the least with propagation. This results to an important finding that vortex rings can dissipate at a faster rate when it is completely opposed rather than a partial objection.

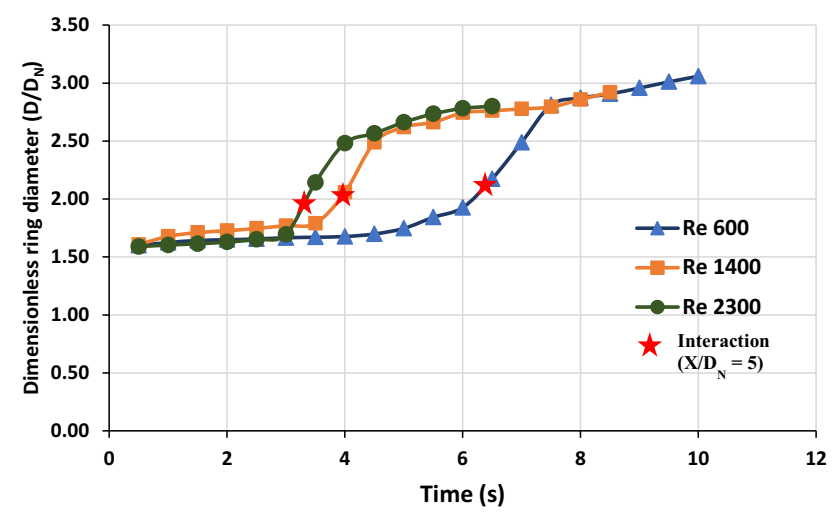

Figure 15. Variation of vortex ring diameter along its propagation during the interaction with the flat surface at different Reynolds numbers.

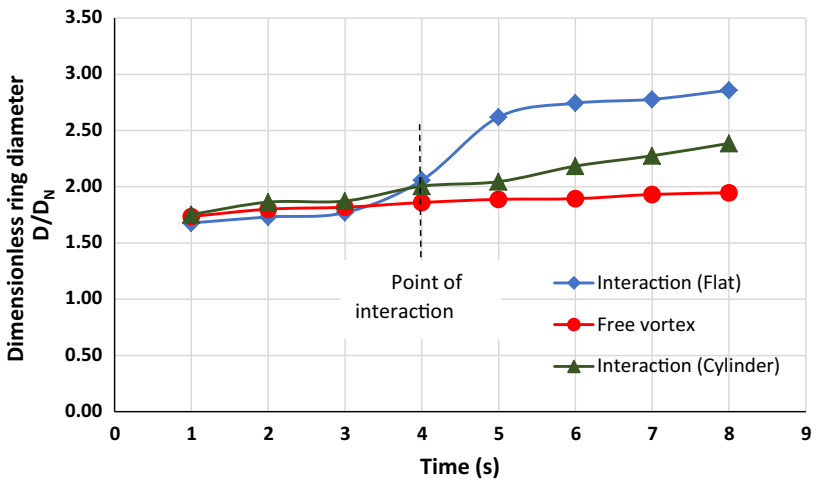

Figure 16. Variation of vortex ring diameter along its propagation for free vortex, interaction with flat surface and cylinders at $L_{N} / D_{N}=1$ and $R e=1400$.

\section{Conclusion}

The vortex ring characteristics are studied during its interaction with the solid bodies, namely the circular cylinder and flat surface. The vortex rings generated with different stroke lengths and velocity is characterized for its ring diameter, velocity, and reconnections after the interaction. The vortex rings generated with the stroke length ratios of more than 4 has trails following the vortex ring. The velocity of a vortex ring is reduced upon propagation, and its diameter is seen to increase. Results indicate that the vortex ring diameter depends on the stroke length ratio at which the fluid is ejected. However, the vortex core diameter is a function of both stroke length and Reynolds number.

The interaction results of vortex rings with different diameter cylinders indicate that for lower diameter cylinders of 0.2 and $0.6 \mathrm{~mm}$, the vortex rings get separated due to the cylinder and instantaneously reconnection is observed after passing over the cylinder. Counter-rotating secondary vortices are observed during the interaction. For large diameter cylinders of 1.5 and $2.5 \mathrm{~mm}$, the deformation of the vortex core is observed, and vortex ring dissipated without reconnection. Vortex ring interaction with flat solid surfaces illustrates the generation of a secondary vortex ring, and rebounding of primary vortex ring that is seen to largely depend on the Reynolds number. Comparing the vortex ring interactions with the cylindrical and flat surfaces, the vortex ring diameter is seen to increase at a larger rate in the latter than that of the former case. Further, vortex rings diffuse at a faster rate when opposed entirely by a solid surface rather than a partial objection like cylinders.

\section{References}

[1] Didden N 1979 On the Formation of Vortex Rings: Rollingup and Production of Circulation, J. Appl. Math. Phys. (ZAMP) 30: 101-116 
[2] Maxworthy T 1972 The structure and stability of vortex rings. J. Fluid. Mech. 51: 15-32

[3] Gharib M, Rambod E and Shariff K 1998 A universal time scale for vortex ring formation. J. Fluid Mech. 360: 121-40

[4] Dabiri J O, Colin S P, Costello A H and Gharib M 2005 Flow patterns generated by oblate medusan jellyfish: field measurements and laboratory analyze. J. Exp. Biol. 208: 1257-1265

[5] Maxworthy T 1977 Some experimental studies of vortex rings. J. Fluid Mech. 81: 465-495

[6] Saffman P G 1970 The velocity of viscous vortex rings. Stud. Appl. Math. 49: 371-380

[7] Lim T T and Nickels T B 1995 Vortex rings. In: Fluid Vortices. Fluid Mechanics and Its Applications (ed. Green, S. I.), vol. 30. Dordrecht: Springer, pp. 95-153

[8] Naitoh T, Okura N, Gotoh T and Kato Y 2014 On the evolution of vortex rings with swirl. Phys. Fluids 26: 067101

[9] Advaith S, Manu K V, Tinaikar A, Chetia U K and Basu S 2017 Interaction of vortex ring with a stratified finite thickness interface. Phys. Fluids 29: 093602

[10] Naitoh T, Sun B and Yamada H 1995 A vortex ring traveling across a thin circular cylinder. Fluid Dyn. Res. 15: 43-56

[11] Ashurst W M T and Meiron D I 1987 Numerical study of vortex reconnection. Phys. Rev. Lett. 58: 1632-1635

[12] Saffman P G 1990 A model of vortex reconnection. J. Fluid. Mech. 212: 395-402

[13] Debopam Das, Akash Manghnani, Mohit Bansal and Prafulla Sohoni 2016 Axial interaction of a vortex ring with a cylinder. J. Fluid Mech. 809:1-30

[14] Kontis K, An R, Kounadis D and Zare-Behtash H 2008 Head-on collision of shock wave induced vortices with a cylinder and a sphere. Int. J. Heat Fluid Fl. 29: 1380-1392
[15] Ren H, Zhang G X and Guan H S 2015 Three-dimensional numerical simulation of a vortex ring impinging on a circular cylinder. Fluid Dyn. Res. 47: 025507

[16] Musta M N 2016 Interaction of a vortex ring with a cutting thin plate. Measurement 88: 104-112

[17] Yamada H, Kohsaka T, Yamabe H and Matsui T 1982 Flow field produced by a vortex ring near a plane wall. J. Phys. Soc. Jpn. 51: 1663-1670

[18] Lim T T, Nickels T B and Chong M S 1991 A note on the cause of rebound in the head-on collision of a vortex ring with a wall. Exp. Fluids 12: 41-48

[19] Walker J D A, Smith C R, Cerra A W and Doligalski T L 1987 The impact of a vortex ring on a wall. J. Fluid Mech. 181: 99-140

[20] Cerra A W and Smith C R 1983 Experimental observations of vortex ring interaction with the fluid adjacent to a surface. Technical Report FM-4. Bethlhem: Lehigh University

[21] Lim T T 1986 A visual study vortex ring/wall interaction. In: 9th Australasian Fluid Mechanics Conference, Auckland. 101-104

[22] Mujal-Colilles A, Dalziel S B and Bateman A 2015 Vortex rings impinging on permeable boundaries. Phys. Fluids 27: 015106

[23] New T H, Shi S X and Zang B 2016 Some observations on vortex-ring collisions upon inclined surfaces. Exp. Fluids 57: 109

[24] Xu Y and Wang J J 2016 Flow structure evolution for laminar vortex rings impinging onto a fixed solid wall. Exp. Therm. Fluid Sci. 75: 211-219

[25] Adhikari D 2009 Some experimental studies on vortex ring formation and interaction. Master's thesis, National University of Singapore. http://scholarbank.nus.edu.sg/han dle/10635/16569 